Proceedings of XIX International Scientific Conference "New Technologies and Achievements in Metallurgy, Material Engineering, Production Engineering and Physics", Częstochowa, Poland, June 7-8, 2018

\title{
The Effect of Twilight Myopia Based on Modified Numerical Eye Model
}

\author{
M. Sroga ${ }^{a}$, M. Dośpią $^{a, *}$, A. Prusiński ${ }^{b}$, M. Stala $^{a}$ And R. MAtusiak ${ }^{a}$
}

${ }^{a}$ Częstochowa University of Technology, Faculty of Production Engineering and Materials Technology,

Institute of Physics, al. Armii Krajowej 19, 42-200 Częstochowa, Poland

${ }^{b}$ Research and Development Centre for Mechanical Appliances OBRUM Ltd, Toszecka 102, 44-117 Gliwice, Poland

To determine the effect of twilight myopia on retinal quality image and to explain the Purkinje effect, the numerical model of Gullstrand's eye was modified. Modification was concerning on addition of the iris in vision path and chromatic properties for the optical elements of modeled eye. Applied changes allowed to simulate propagation of light rays under photopic and mesopic vision conditions. The obtained results were used to simulate retinal spot diagrams, during day and under low light intensity, as in the case of twilight.

DOI: 10.12693/APhysPolA.135.169

PACS/topics: anatomy and optics of eye; vision: light detection, adaptation, and discrimination; wave propagation in random media; image forming and processing

\section{Introduction}

In recent years, intensive research on personalization and adaptation to environmental conditions of an eye refraction correction, has been performed. Among the studies in the scope of personalization of the visual system correction, one can mention e.g. research on the inclusion in the construction of progressive glasses of such factors as disparity vergence or phoria and many other [1]. In the case of adapting to specific environmental conditions, there can be distinguished several leading paths, such as works on progressive glasses for children in order to stop the myopia epidemic [2,3], eye glasses dedicated for driving vehicles under mesopic conditions $[4,5]$ or special lean coatings, protecting the eye from excessive exposure to blue light [6].

This paper is focused on analyzing the effects lying under miopic refraction shift which is correlating with studies performed on eye glasses dedicated for driving vehicles under mesopic conditions.

In order to perform quantitative analysis of that effect, the Gullstrand eye numerical model was modified.

\section{Methodology}

\subsection{The Gullstrand eye numerical model}

According to the Gullstrand model, the eye is built of 5 refractive areas which creates 7 refractive surfaces. The cornea of the eye consists of two surfaces, and a lens of four (two per nucleus and a capsule). The model has two states of accommodation: $0 \mathrm{D}$ - relaxed and $10.87 \mathrm{D}$ - accommodated, but the presence of an iris, as well as chromatic properties of refractive areas, are not described

*corresponding author; e-mail: mdospial@wp.pl in formalism. There exists also a simplified version of the Gullstrand eye model, but it will not be discussed here. Below, in Table I, are gathered parameters describing presented model [7].

TABLE I

Parameters describing Gullstrand schematic eye [7].

\begin{tabular}{|c|c|c|}
\hline Parameter & Relaxed & Accommodated \\
\hline \multicolumn{3}{|c|}{ Index $n$} \\
\hline cornea & 1.376 & 1.376 \\
\hline anterior chamber & 1.336 & 1.336 \\
\hline lens capsule & 1.386 & 1.386 \\
\hline crystalline lens & 1.406 & 1.406 \\
\hline vitreous humor & 1.336 & 1.336 \\
\hline \multicolumn{3}{|c|}{ Radius $r$ [mm] } \\
\hline cornea & 7.7 & 7.7 \\
\hline anterior chamber & 6.8 & 6.8 \\
\hline front lens capsule & 10 & 5.33 \\
\hline crystalline lens & 7.911 & 2.655 \\
\hline rear lens capsule & -5.76 & -2.655 \\
\hline vitreous humor & -6.0 & -5.33 \\
\hline retina & -17.2 & -17.2 \\
\hline \multicolumn{3}{|c|}{ Thickness [mm] } \\
\hline cornea & 0.50 & 0.50 \\
\hline anterior chamber & 3.10 & 2.7 \\
\hline front lens capsule & 0.546 & 0.6725 \\
\hline crystalline lens & 2.419 & 2.655 \\
\hline rear lens capsule & 0.635 & 0.6725 \\
\hline vitreous humor & 17.185 & 16.80 \\
\hline \multicolumn{3}{|c|}{ Focal length [mm] } \\
\hline object & -17.055 & -14.169 \\
\hline image & 22.785 & 18.930 \\
\hline \multicolumn{3}{|c|}{ Refractive power [dpt] } \\
\hline cornea & 43.053 & 43.053 \\
\hline crystalline lens & 19.11 & 33.06 \\
\hline schematic eye & 58.636 & 70.57 \\
\hline
\end{tabular}




\subsection{Modifications of numerical model}

In order to describe more complex vision processes, like myopic shift or the Purkinje effect, the Gullstrand eye was modified.

Changes were concerning on addition of the iris in vision path and chromatic properties for the optical elements. The pupil in vision path was added at a distance of $3.2 \mathrm{~mm}$ from the front apex of the cornea, which corresponds to the edge of the front of the lens when it is maximally accommodated. Basing on [8], the diameter of the iris during photopic light conditions was assumed to be equal to $3 \mathrm{~mm}$ and in case mesopic, $6 \mathrm{~mm}$.

The chromatic properties - wavelength dependent index and the Abbe number of all optical elements - were described basing on Liou et al. as well as Chauuhuri and Lakshminaiiay $[9,10]$.

Performed changes in chromaticity of schematic eye elements as well as addition of the iris in vision path (of $3 \mathrm{~mm}$ diameter), resulted in modification of vitreous body thickness. All parameters for relaxed eye after changes are gathered in Table II.

Parameters describing modified Gullstrand schematic eye.

TABLE II

\begin{tabular}{l|c|c|c|c|c|c}
\hline \hline \multirow{2}{*}{ Parameter } & \multirow{2}{*}{ Radius $r[\mathrm{~mm}]$} & \multirow{2}{*}{ Distance $[\mathrm{mm}]$} & \multicolumn{3}{|c|}{ Index $n$} & \multirow{2}{*}{ Abbe number } \\
\cline { 4 - 6 } & & $656.3 \mathrm{~nm}$ & $587.6 \mathrm{~nm}$ & $486.1 \mathrm{~nm}$ & \\
\hline cornea & 7.70 & 0 & 1.380 & 1.383 & 1.387 & 52.64 \\
anterior chamber & 6.80 & 0.5 & 1.331 & 1.334 & 1.337 & 56.43 \\
front lens capsule & 10.00 & & 1.388 & 1.390 & 1.394 & 62.41 \\
crystalline lens & 10.00 & 3.6 & 1.408 & 1.410 & 1.414 & 69.04 \\
rear lens capsule & 7.91 & 4.146 & 1.388 & 1.390 & 1.394 & 62.41 \\
vitreous humor & -5.76 & 7.565 & 1.331 & 1.334 & 1.337 & 56.43 \\
retina & -6.00 & & & & &
\end{tabular}

\section{Results and discussion}

Figure 1a presents eye model with diaphragm (iris) of $3 \mathrm{~mm}$ diameter, and corrected thickness of vitreous body, in respect to chromaticity of optical elements. The figure also shows the traces of rays through the optical system of the modeled eye, where focal point of that system lies exactly on retina.

In Fig. 1b there are presented spot diagrams for retinal image (central plot) and defocused by $1 \mathrm{~mm}$ and $2 \mathrm{~mm}$ in longitude axis in both directions. The diameters of spots, in defocused spot diagrams, were used to determine the position of the smallest blur spot, and calibration of the model. Precisely, they were used to apply the change in vitreous body thickness and position of the retina, so that the focal point lies precisely on it. The diameter of the smallest blur spot illustrates the value of spherical aberration, and is equal to $0.016 \mathrm{~mm}$.

Figure $2 \mathrm{a}$ presents eye model after changing the diaphragm size (iris) to $6 \mathrm{~mm}$. The figure also shows the traces of rays in such defined eye, as well as value and direction of the focal point shift. Obtained defocus towards the front of the eye was equal to $0.72 \mathrm{~mm}$. This corresponds to shifting the eye to myopia by the value of $2.88 \mathrm{D}$. This result differs from the literature data. The largest value of the ocular myopic shift was equal to $0.75 \mathrm{D}$ [11]. One of the explanations regarding the existing difference is shown to us by the analysis of spot diagrams (Fig. 2b).
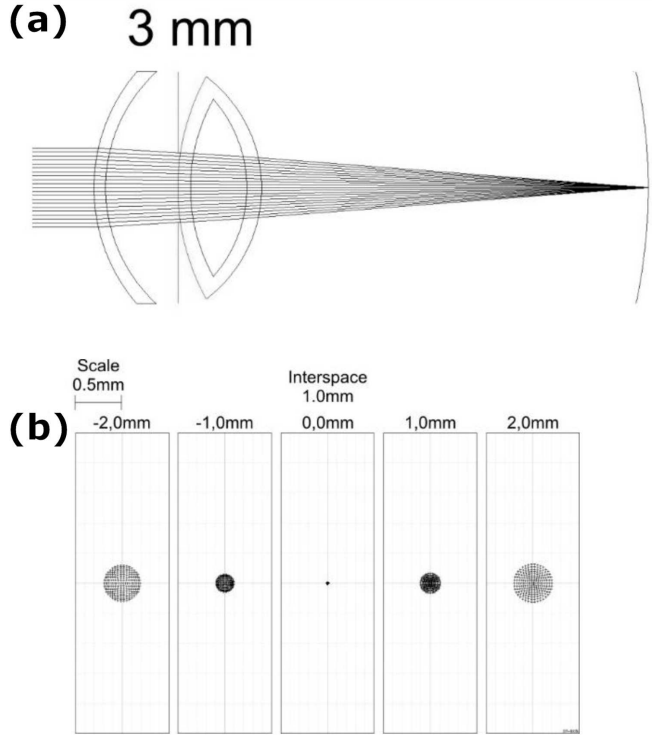

Fig. 1. (a) Modified schematic eye model with diaphragm diameter of $3 \mathrm{~mm}$, with marked ray trace, (b) direct spot diagrams of retinal image, and after its defocus by the marked value.

The size of the smallest blur spot, for the eye with a diaphragm diameter of $6 \mathrm{~mm}$, was $0.22 \mathrm{~mm}$, which gave 13.75 times the value of that parameter as for the eye with a diaphragm of $3 \mathrm{~mm}$ and was defocused towards 


\section{(a) $6 \mathrm{~mm}$}
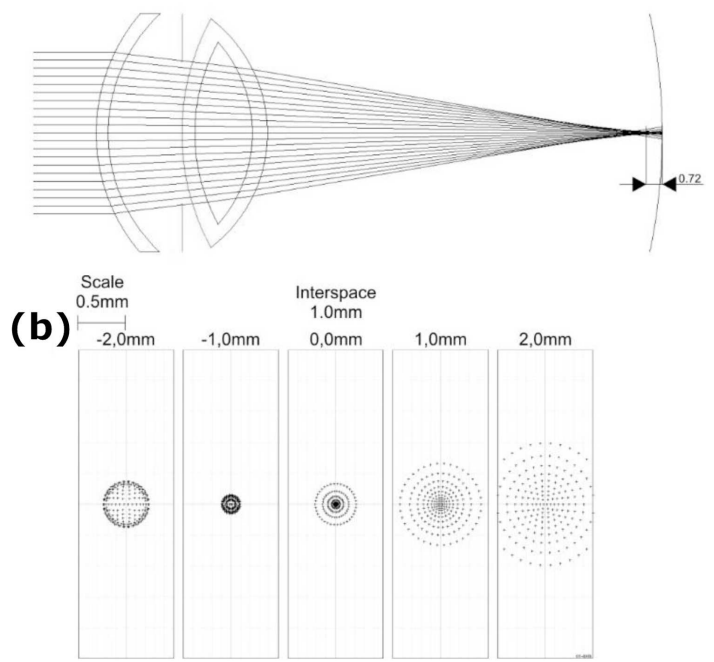

Fig. 2. As in Fig. 1, but for diaphragm diameter of $6 \mathrm{~mm}$.

the front of the eye. This shows a significant spherical aberration of the model, and that is where we see a discrepancy in the power range in case of shifting the eye to myopia.

The obtained results have been additionally corrected by the shift resulting from the Purkinje effect, which is related with the shift of the focal point of the eye, from the yellow to red focus. This is manifested in the fact that with the dilated pupil, during the twilight photometric conditions, the observed image is reddened. The correction was determined by changing the wavelength while analyzing the course of rays through the model. The obtained focal point defocus, corresponded to the change in the power of the system by $0.2 \mathrm{D}$. In addition, it was assumed that the lights illuminate the area in front of the car while driving, but to a limited extent. It has been assumed that the focus point of sight is located at a finite distance, i.e. around $3 \mathrm{~m}$. This corresponds to correction of an optic system power for about $0.33 \mathrm{D}$.

\section{Conclusions}

The performed modifications to the Gullstrand model, in the form of added pupil of the eye and the chromaticity of the optical elements, allowed to simulate the twilight myopia and the Purkinje effect.

Numerical calculations of physiological changes induced by adaptation to environmental conditions during driving under twilight view state, allowed to determine the quantitative limits of the observed defocus effect. The size of the defocus was compared with the experimental data measured by Lopez-Gil et al. [11]. Differences in the value of image defocus, for theoretical calculations $(2.88 \mathrm{D})$ and experimental measurements $(0.75 \mathrm{D})$, have been attributed to the imperfections of the Gullstrand eye model, in which all optically active surfaces are spherical. This translates into a large spherical aberration of the optical system, which in turn results in a significant shift of the spot of the slightest blur towards myopia, for the wide open pupil. In the real eye, both the corneal surfaces and the lenses have an aspherical topography, which significantly reduces spherical aberration [12]. Additionally during driving the observed object lies in a finite distance and our eye starts to use instead of yellow - the red focal point (the Purkinje effect), these conditions reduce the displacement effect (to $2.35 \mathrm{D}$ ), however, final results are still not equated with the experimental ones. Obtained results unambiguously indicate the applicability of correction in the form of diffusing glasses for twilight driving, however, the discrepancy between experimental and theoretical data indicates that further studies of this effect are needed on more sophisticated models, which additionally are taking into account the asphericity of the different surfaces of the eye.

Based on the obtained results, it also can be concluded that the modified model can be used perfectly to explain the observed effects of twilight myopia and the Purkinje effect in a simple way.

It should be also mentioned that described case (eyeglasses reducing twilight myopic shift during driving) is not the only practical application of performed simulations. Developed changes, in terms of the myopic shift, related with pupil size changes, can be also used to modify the autorefractometer programs by adding a test option correlating with use of the tropicamide. Such a study would give information about correction of power resulting from pupil size changes after palsy, which would make the result of the research more real.

\section{References}

[1] T.L. Alvarez, E.H. Kim, B. Granger-Donetti, Sci. Rep. 7, 2529 (2017).

[2] B. Drobe, C. Carimalo, US Patent US9791718B2 (2017).

[3] J.M. González-Méijome, EBioMedicine 16, 24 (2017).

[4] N. López-Gil, S.C. Peixoto-de-Matos, L.N. Thibos, J.M. González-Méijome, J. Vision 12, 4 (2012).

[5] Xin Ping Yu, Jinhua Bao, B. Drobe, Wehui Lv, Hao Chen, Jinling $\mathrm{Xu}, \mathrm{Ge} \mathrm{Wu}$, US Patent US20170023798A1 (2017).

[6] UV and Blue-Violet Light Definitions, risks and prevention, Collection of articles from 2011 to 2015, Essilor International, 2016.

[7] A.H. Tunnacliffe, Introduction to Visual Optics, 1989.

[8] Feng Rao, A.H.S. Chan, Xi-Fang Zhu, Vision Research 137, 24 (2017).

[9] H. Liou, N. Brennan, J. Opt. Soc. Am. A 14, 1684 (1997).

[10] A. Chauuhuri, V. Lakshminaiiay, J. Indian Inst. Sci. 66, 307 (1986).

[11] N. Lopez-Gil, S.C. Peixoto-de-Matos, L.N. Thibos, J.M. González Méijome, J. Vision 12, 1 (2012).

[12] R. Navarro, J. Santamaria, J. Bescos, J. Opt. Soc. Am. A 2, 1273 (1985). 